\title{
Two minutes Talk Therapy (TTT): A tool to improve medication adherence of
} Sri Lankan hypertensives.

\section{Kumanan $T^{1}$}

${ }^{1}$ Department Of Medicine, Faculty of Medicine, Jaffna,

\section{Introduction}

Hypertension is the most important risk factor for the commonest causes of death in this country; namely coronary vascular disease, stroke, heart and renal failure. Nearly one-fifth to one-third of adult Sri Lankan population have blood pressure levels that are above normal and the prevalence is comparable to those of the western world $(1,2,3)$. Though Sri Lanka has immensely benefitted through free health delivery system, the nation has been facing challenges in combating the silent killer non-communicable diseases. Non-adherence of antihypertensive medication is a serious public health issue of the country (4). Only few studies have looked into the reason for noncompliance and it has been found that although the knowledge about hypertension is reasonably good among the hypertensives, forgetfulness and interruptions of daily routine were identified as common reasons for nonadherence (5). Over time, several strategies have been analyzed and postulated by researchers to improve medication adherence of hypertensive patients. Of these strategies, educating and enlightening the patient on hypertension and its treatment by the primary care physician during the initial encounter appears to be very promising. This initial talk therapy is often overlooked by most physicians working under poor resource settings. This article highlights the importance of such brief encounters and the content to be addressed in such encounters so that this golden opportunity of a contact with the patient is not missed.

\section{The importance of communication skills}

Communication skills in medical care is highly correlated with better medication adherence, and training physicians to enhance their communication skills with their patients would result in better medication adherence. This skill must be incorporated into medical education programs for both under and postgraduates. Interventions to improve treatment adherence, supporting arguments that communication is important and resources devoted to improving clinical communication skills are worth investing in and would be cost effective in managing chronic illnesses. Communication is thus an important factor over which physicians have some direct control in helping their patients to improve medication adherence (6).

\section{Reasons for medication nonadherence in Sri Lankans}

Amulti-center study among South Asian population recently concluded that more than half of the hypertensives of Sri Lankan rural population have not achieved the target blood pressure levels and warranted urgent intervention to improve medication adherence (7).

Only few studies to date tried to explore the reasons for noncompliance among hypertensive patients in Sri Lanka. A recent study from Northern Sri Lanka concluded that the most common reasons for nonadherence were forgetfulness $(23.1 \%)$ and interruptions due to daily routines $(17.5 \%)(8)$.

\section{Methods employed to improve compliance}

Motivational interviewing (MI) has increasingly being recognized as a tool to improve medication adherence in the recent past (9). Similarly, brief cognitive behavior therapy (CBT) is also recognized as an effective method to impart knowledge and 
importance of medication adherence in patients with chronic medical ailments (10). Employing these concepts to design the brief clinical consultation should enhance medication adherence in patients with hypertension.

\section{A proposed model of 2 min talk therapy}

The consultation should ideally be held in a quiet environment away from unnecessary interruptions. The conversation should be conducted in a compassionate and caring manner using language that the patient can understand, avoiding complicated medical jargon. The following is the schematic flow of the conversation that should be conducted with the patient, when a clinical decision has already been made to initiate pharmacotherapy and allowing the patient to ask questions during the conversation to seek any clarification.

i) You have got high blood pressure. This has been confirmed through repeat BP recordings and is definitive. (Important to remove any doubt from the mind of the patient)

ii) Do you know what the "normal value" should be for Blood Pressure?. (Respond by giving the normal value for Systolic and Diastolic $\mathrm{BP}$ readings and emphasize the importance of BOTH values)

iii) High Blood Pressure can only be CONTROLLED and cannot be cured. (Explain that it is a LIFE-LONG condition)

iv) Ask what will happen if the high BP is left untreated. (Explain using a pictogram of the consequences viz Target Organ Damage)

v) Explain that the BP can only be controlled by Life-style changes (tailor this to the patient as appropriate) AND by taking one or more medications.

vi) Ask the patient's view on whether these medications would harm or be of benefit? Emphasize that the medications are very safe BUT may have some minor side effects. (List the common side effects of the medications). Side effects also mean that the medications are working. vii) If the patient does develop any of the side effects, they should let the doctor know during the next clinic visit. Indicate that if the side effects are troubling them, alternative medications are available. THEY SHOULD NEVER STOP THE MEDICATIONS without informing the doctor.

viii) Ask the patient to pick a time(s) of the day when they will always take the medications and agree with a loved one to remind them daily whether the medication has been taken.

In summary, enlightening the knowledge of patients with hypertension by the treating physician during the initial encounter by spending two minutes of their invaluable time would minimize non-adherence of antihypertensive medications and results in better outcome. The clinicians should be prepared and rehearsed to deliver information in a schematic and compassionate manner within few minutes, avoiding medical jargon at a suitable environment. In such way the primary care physicians of this country could play a pivotal role in combating the silent killer disease and its devastating consequences.

\section{References}

1. Wijewardene K, Mohideen MR, Mendis S, et al. Prevalence of hypertension, diabetes and obesity: baseline findings of a population based survey in four provinces in Sri Lanka. Ceylon Med J. 2005 Jun;50(2):62-70. PubMed PMID: 16114771.

2. Katulanda P, Ranasinghe P, Jayawardena R, Constantine GR, Rezvi Sheriff MH, Matthews DR. The prevalence, predictors and associations of hypertension in Sri Lanka: a cross-sectional population based national survey. Clin Exp Hypertens. 2014 Nov 1;36(7):484-91.

3. Kasturiratne, A., Pinidiyapathirage, M.J., Pathmeswaran, A., et al. (2011) Epidemiology of Hypertension in an Urban Population of Sri Lanka. Ceylon Medical Journal, 65, 256.

4. Kumanan T, Guruparan M, Mohideen MR. Non-adherence of antihypertensive therapy:

Jaffna Medical Journal 
A serious public health issue in Sri Lanka. Journal of the Ceylon College of Physicians. 2016;47(1):50-1.DOI: http://doi.org/10.4038/ jccp.v47i1.7772

5. Pirasath S, Kumanan T, Guruparan M. A study on knowledge, awareness, and medication adherence in patients with hypertension from a tertiary care centre from northern Sri Lanka. Int J Hypertens. 2017;9656450. Article ID 9656450, 6 pages, 2017. https://doi. org/10.1155/2017/9656450.

6. Zolnierek KB, Dimatteo MR. Physician communication and patient adherence to treatment: a meta-analysis. Med Care. 2009;47(8):826-834.

7. Jafar TH, Gandhi M, Jehan I, Naheed A, de Silva HA, Shahab H, et al. Determinants of Uncontrolled Hypertension in Rural
Communities in South Asia-Bangladesh, Pakistan, and Sri Lanka. Am J Hypertens. 2018 Apr 26;31(11):1205-14.

8. Silveira LCJ, Aliti GB, Da Silva EM, Pimentel RP, Gus M, Rabelo-Silva ER. Effect of motivational interviewing in hypertensive patients (MIdNIgHT): study protocol for a randomized controlled trial. Trials. 2019;20(1):414. Published 2019 Jul 9. doi:10.1186/s13063-019-3486-1

9. Moreno B M, Contreras R D, Martínez S N, Araya G P, Livacic-Rojas P, Vera-Villarro P. Evaluación del efecto de una intervención cognitivo-conductual sobre los niveles de presión arterial en adultos mayores hipertensos bajo tratamiento médico . Vol. 134, Revista médica de Chile . scielocl ; 2006. p. 433-40. 